\title{
Taking cues from nature: Hemoglobin catalysed oxygen reduction
}

\author{
Stanislav V. Sokolov, Lior Sepunaru, Richard G. Compton*
}

\begin{abstract}
We describe experiments which elucidate the reaction mechanism of electrochemical oxygen reduction reaction $(\mathrm{ORR})$ on a glassy carbon electrode modified with hemoglobin in an aqueous environment but with the hemoglobin physically separated from the electrode by a porous Nafion layer. Catalytic amplification of the current signal from the reduction of oxygen in a first electron transfer step is observed in the presence of hemoglobin immobilized within a Nafion coating. An overall four electron ORR mechanism is inferred and validated.
\end{abstract}

\section{Introduction}

As the production of renewable energy must rise there is a growing need for the storage of the generated energy. Electrochemical approaches are an attractive option due to scalability, large capacity and potentially low cost. [1] The oxygen reduction reaction in particular is of immense technological importance for the development of fuel cells. [2,3] However the slow kinetics of reduction on carbon electrodes is a major limitation to its practical adoption and application. Typically platinum has been employed as a cathode catalyst in order to overcome the kinetic limitations but leads to higher costs and potential long term unsustainability. As a result a search for alternative catalysts is warranted. Hemoglobin is known for its interactions with oxygen and as a result is a suitable potential candidate catalyst.

The schematic structure of the hemoglobin protein is shown in Figure 1. It consists of four protein subunits, each with a redox active Fe centre, which is mainly responsible for the binding of oxygen [4]. Each subunit is arranged mostly in an alpha helical structure. The surrounding protein structures serve to 'protect' the redox centre against potentially fatal $\mathrm{CO}$ and $\mathrm{CO}_{2}$ binding to the Fe redox centre [5] and also supports the cooperative oxygen binding, as discussed thoroughly in the literature. [4] The redox active centre heme, can be extracted from Hemoglobin and in the oxidized form is known as Hemin.

\footnotetext{
*Department of Chemistry,Oxford University Physical and Theoretical Chemistry Laboratory, South Parks Road,Oxford, OX1 3QZ (UK) E-mail:richard.compton@chem.ox.ac.uk
} 


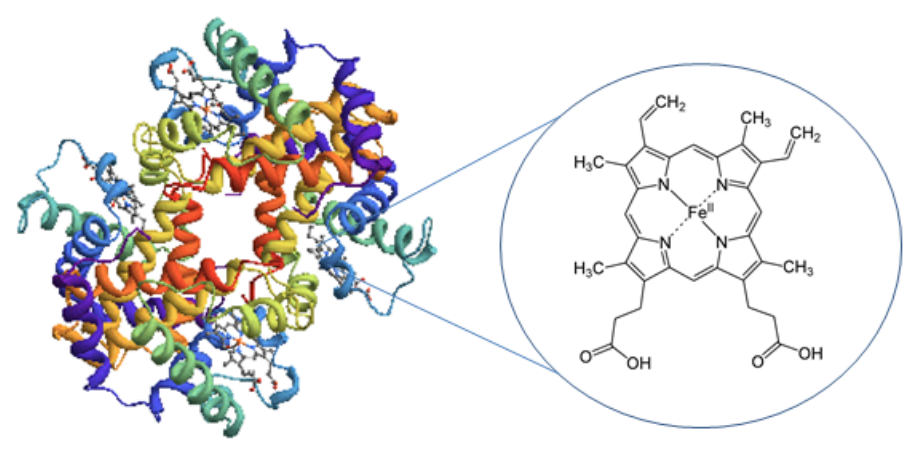

Figure 1: Schematic structure of hemoglobin showing four protein subunits and the inset shows the active Fe (heme site). (Structure source: Wolfram Mathematica 10.4)

There is significant controversy regarding the actual oxidation state of iron, when bound to dioxygen. The oxygenated form of hemoglobin (oxy-Hb) is known to be diamagnetic, while dioxygen and iron (Fe(II)) are both paramagnetic. Currently accepted models suggest that the oxidation state of $\mathrm{Fe}$ is +III. [6] The literature contains several reports of direct Fe-Hb electrochemistry generally made via different forms of electrode modification. Unmodified electrodes generally show poor response towards hemoglobin and a wide range modifications have been adopted ranging from relatively simple cystine modifications [7] to complex multi-layered structures. [8]. This observation could possibly be explained by the efficient shielding of the redox centre by the surrounding proteins and as a result direct electron transfer is hindered. [9] So despite over a hundred publications (see Section 1.1) little progress has been made towards the understanding of the electrochemistry of hemoglobin or practical applications towards sensor development. [9] The main debate stems from whether the Fe core is accessible for direct electrochemical redox activity and the results appear to be highly electrode-sensitive. [7] We distinguish two approaches to hemoglobin electrochemistry: direct electron transfer with the Fe core and indirect interaction of oxygen with $\mathrm{Hb}$ within close proximity to the electrode and focus on the indirect path as a means to catalyse the oxygen reduction reaction. In the following work we modify the electrode using a procedure developed Pumera et. al [10] in which the $\mathrm{Hb}$ is physically 'sandwiched' between two layers of oxygen permeable Nafion so as to eliminate the possibility of direct electron transfer between the electrode and $\mathrm{Hb}$ and allow us to study the effect of $\mathrm{Hb}$ on the oxygen reduction reaction itself.

In general the use of electrochemistry, more specifically voltammetry to study the hemoglobin/ oxygen system whilst intrinsically sensitive and qualitative, presents a significant challenge when applied in practice. These difficulties arise primarily from two sources. First the reaction is a multistep process involving a sequence of electron transfer steps, chemical reactions and creating several chemical intermediates of differing lifetimes. Second the layers of Nafion supported hemoglobin on the electrode surface display significant and significant levels of porosity, so that the mass transport of the reactants, intermediates and products does not follow the simple semi-infinite diffusive character of flat, planar surfaces, which is widely used to generate the basic equations employed in analysing much voltammetry. The above points can be well illustrated by the study of the oxygen reduction using so-called Koutecky-Levich (K-L) analysis of data obtained at a rotating disk electrode. This requires the measurement of a current-voltage curve as a function of the electrode rotation speed and an extrapolation of the transport limited current to induce rotation speed to overcome (semi-infinite) diffusion effects. The method was introduced to analyse a single one-electron transfer coupled with a preceding first order chemical step. Although Koutecky-Levich analysis has become widely used to study the ORR this is considered as unreliable since the underpinning theoretical analysis does not embrace multi-step processes including intermediates of a finite lifetime. This was first clarified by Johnson and co-workers [11] in a much cited paper and more recently re-emphasised by Qiao 
Table 1: Range of hemoglobin complexes oxidation states with biological significance

\begin{tabular}{l|l|l}
\hline Hemoglobin Complex Active Centre & Name & \multicolumn{2}{l}{ Biological function } \\
\hline $\mathrm{Fe}(I I)$ & Hemoglobin & Able to bind oxygen (active form) \\
$\mathrm{Fe}(I I I)$ & Methemoglobin & Unable to bind oxygen (Inactive form) \\
$\mathrm{Fe}(I I I) \mathrm{O}_{2}^{-}$ & Oxy-hemoglobin & Hemoglobin with bound oxygen, oxygen transport mechanism \\
$\mathrm{Fe}(I V) \mathrm{O}$ & Ferryl-hemoglobin & Oxidized form of hemoglobin, by-product of cell reactions \\
$\mathrm{Fe}(I V) \mathrm{O}$ & Oxo-ferryl Hb & Reactive form of Hb with radical in the globin group
\end{tabular}

and colleagues in ACS Catalysis. [12] Further the K-L analysis has been theoretically shown to be inapplicable to rough and/or porous surfaces. [13] In order to study the hemoglobin/oxygen system alternative voltammetric approaches are needed. A second approach commonly, but questionably, applied to the ORR is the use of a double working electrodes (generator-collector) systems most commonly in the form of rotating-ring disc electrode (RRDE) as introduced by Frumkin and Nekrasov [14] in Moscow, although other geometries (such as channels or wall-jets) have been occasionally used. In this method the current signals on a second electrode are used to infer information about the process taking place on the first electrode with a knowledge of the convective-diffusive regime linking the two electrodes. [15] In the context of the ORR the RRDE has been used to distinguish between a two and a four electron transfer process which would respectively lead to the formation of either hydrogen peroxide $\left(\mathrm{H}_{2} \mathrm{O}_{2}\right)$ or water $\left(\mathrm{H}_{2} \mathrm{O}\right)$. The RRDE setup allows these products to be distinguished under some conditions using so-called collection efficiency measurements but again the multistep nature of the reaction on both the generator and collector electrodes leads to significant complications in many cases as fully summarized by Qiao. [12] The latter explicitly conclude that platinum collector electrodes are unreliable, but with a knowledge of the likely reaction pathway, concluded that in alkaline media, at least, gold (ring) collector electrodes may be reliable. Nonetheless their data analysis presumes semi-infinite diffusion-convection at the disc (generator) electrode so that if the latter is modified with a porous surface layer the reliability of the RRDE method is also in question. Most generally Qiao et al. state future studies are extremely desirable to develop a correct and accurate method [to detect the number of electrons transferred]. In the present paper we attempt to accept this challenge, eschewing the use of double electrode, convective systems but driven by the desire to develop a relatively simple method, which does not require the construction of elaborate electrode systems or the use of flowing solutions. To this end we show how cyclic voltammetry allied with a knowledge of solubilities, diffusion coefficients and chemical reactivities together with Tafel analysis can be used to powerful effect in the analysis of electrodes modified with porous layers which include coupled catalytic activity.

An innovative,powerful approach introduced by Pumera et al. [10] is applied and a mechanism is proposed for the catalytic oxygen reduction reaction. By using the Nafion 'sandwich structure' we physically separate Hemoglobin from the electrode surface in order to simplify the system and investigate the reduction of oxygen in the presence of hemoglobin, rather than the direct Hb electrochemistry which has been reported previously.

\subsection{Electrochemistry of hemoglobin}

In this section we overview previous work on hemoglobin electrochemistry and a brief discussion of pathways of electrochemical oxygen reduction at glassy carbon electrodes.

\subsubsection{Oxidation states of hemoglobin}

Table 1 summarizes the oxidation states of Fe redox centre commonly encountered experimentally and of biological relevance. It is known that hemoglobin by itself without bound oxygen can have $\mathrm{Fe}(\mathrm{III})$ or $\mathrm{Fe}(\mathrm{II})$ oxidation states. [16] The Fe(III) form is known as methemoglobin and it is unable to bind oxygen. $[17,18]$ The oxygen binding form of hemoglobin has an iron oxidation state of $\mathrm{Fe}(\mathrm{II}) \cdot[19,20]$ The nature of the bonding within the oxy-hemoglobin complex is currently accepted 
to be the $\mathrm{Fe}(\mathrm{III})$ oxidation state combined with a dioxygen radical with a degree of covalent bonding. [20] Other oxidation states are often by-products of hemoglobin decay and reactions with reactive superoxide species within cells. $[21,22]$

\subsubsection{Direct hemoglobin electrochemistry}

A reversible electrochemical response from hemoglobin was claimed by Salimi et al. using a glassy carbon modified with nickel oxide nanoparticles. Two redox peaks were observed voltammetrically. Scan rate vs peak current analysis indicated a surface-confined redox process. The presence of the peaks was attributed to direct electron transfer of hemoglobin with the electrode. [23] Amreen et al. have reported an electrochemically reversible response of red blood cells on a glassy carbon electrode modified with dropcast graphitized mesoporous nanocarbon (GMC) and Nafion. [24]. For the case of GMC modified electrodes the authors have trialed various forms of carbon; however only one specific form of mesoporous nanocarbon produced a reversible response. Most importantly the reverse oxidative peak was only observable with a Nafion layer (Naf) but not on a pure GMC modified electrode, which is indicative of a complex process taking place. In both cases the authors have attributed the observed response to the $\mathrm{Fe}(\mathrm{II}) / \mathrm{Fe}(\mathrm{III})$ redox couple and asserted the need for suitable electrode materials in order to allow direct electron transfer to take place. Additional works include a wide variety of electrode modifications [25-27] and the interested reader is referred to a review by Scheller et al. [9]

\subsubsection{Indirect hemoglobin electrochemistry}

The main biological function of hemoglobin is binding and transportation of oxygen. [28] As a result it is important to study the interaction of $\mathrm{Hb}$ and dioxygen in the solution phase. A suitable method involves monitoring the oxygen reduction reaction at a hemoglobin functionalized electrode and observing the resultant reductive current response. [10] The basis for such a method is described in section 1.2 .

\subsection{Oxygen reduction reaction on glassy carbon electrode (ORR)}

ORR on a glassy carbon electrode typically leads to the formation of hydrogen peroxide involving a two electron transfer (Reaction 1). [2,3,29]

$$
\mathrm{O}_{2}(\mathrm{aq})+2 \mathrm{H}^{+}+2 \mathrm{e}^{-} \longrightarrow \mathrm{H}_{2} \mathrm{O}_{2}
$$

The four electron conversion to water (Reaction 2)is kinetically slow and requires significant overpotential and is generally unfavourable on a glassy carbon electrode. [30,31] As a result this reaction on a glassy carbon can be used as a baseline for the efficiency of oxygen reduction reaction and measurement of subsequent catalytic activity for different electrode modifications.

$$
\mathrm{O}_{2}(\mathrm{aq})+4 \mathrm{H}^{+}+4 \mathrm{e}^{-} \longrightarrow 2 \mathrm{H}_{2} \mathrm{O}
$$

\section{Experimental}

All chemicals were purchased from Sigma Aldrich unless stated otherwise and used without further purification. A $\mu$ Autolab II potentiostat (Metrohm-Autolab BV, Utrecht, The Netherlands) was used to control the electrochemical experiments with the software NOVA 1.10. The electrochemical cell was shielded by a single-wall Faraday cage. A three electrode setup was used with a glassy carbon working electrode (diameter $=3.05 \mathrm{~mm}$ ), a Saturated Calomel Electrode [32] as the reference electrode (0.241 V vs Standard Hydrogen Electrode) and platinum mesh as a counter electrode. The experiments were performed under thermostatted conditions $\left(25.0 \pm 0.1 \mathrm{C}^{\circ}\right)$. Before all experiments 
and between experimental repeats the electrode was polished using a diamond spray (Kemet, Kent, UK) in the size sequence of $3.0 \mu \mathrm{m}, 1.0 \mu \mathrm{m}$ and $0.1 \mu \mathrm{m}$ to a mirror-like finish to ensure clean and reproducible surface before subsequent functionalization. Phosphate saline buffer (PBS) was used for all experiments. PBS buffer was prepared as the following solution; $139.7 \mathrm{mM} \mathrm{KCl}, 10 \mathrm{mM} \mathrm{Na} 2 \mathrm{HPO}_{4}$ and $1.8 \mathrm{mM} \mathrm{KH}_{2} \mathrm{PO}_{4}$. The $\mathrm{pH}$ was adjusted to 7.4 using hydrochloric acid and confirmed using a $\mathrm{pH}$ probe (Hanna Instruments $\mathrm{pH} 213$ meter). UV-vis spectroscopy experiments were conducted in the same buffer media using a Shimadzu spectrometer UV-1800. For optical microscopy a Carl Zeiss Examiner A1 microscope with a 20x air objective was used.

\subsection{Electrode modification}

The electrode was prepared via a modified procedure originally innovated by Pumera et al. [10] The modification was performed using the following general procedure: individual layers (Nafion or hemoglobin) were sequentially dropcast on the glassy carbon electrode and allowed to dry under nitrogen flow. The effect of layer thickness was investigated by individually varying the concentration of Nafion and hemoglobin used in a particular layer. Nafion 117 solution (5\% in a mixture of lower aliphatic alcohols and water) was used for the film preparation. Hemoglobin was dissolved in deionised water and dried under nitrogen gas flow. The optimized procedure involved $2 \mu L$ of Nafion 117 solution ( $5 \%$ in a mixture of lower aliphatic alcohols and water), $10 \mu \mathrm{L}$ of Hb solution $\left(0.002 \mathrm{~g} \mathrm{~mL}^{-1}\right.$ dissolved in deionised water), $4 \mu \mathrm{L}$ of Nafion 117 , dropcast sequentially, leading to layered films,subsequently referred to as Naf-Hb-Naf. This procedure has shown the best hemoglobin immobilization as well as high reproducibility. The Nafion film thickness was estimated to be few microns in accordance with Equation 3.

$$
t=\frac{m}{A \rho}
$$

where $\mathrm{m}$ is the mass of dropcast Nafion, $\mathrm{A}$ is the area of the film, $\rho$ is the approximate density of the film. From that, an estimated thickness of a few microns for each Nafion layer was calculated.

\section{Results}

UV-vis spectroscopy was used to determine the oxidation state of hemoglobin and to provide a starting point for the investigation. The effect of Nafion presence on the ORR was evaluated by adjusting the thickness of the films. Subsequently The Naf-Hb-Naf films were investigated using optical microscopy for evidence of agglomeration and porosity. Having established the stability of the films a series of electrochemical experiments were performed in PBS buffer under air and oxygen atmospheres. The obtained results allowed us to infer the mechanism of oxygen reduction and its interaction with hemoglobin.

\subsection{UV-vis spectroscopy}

In order to determine the oxidation state of hemoglobin solution used for subsequent dropcasting $\left(0.002 \mathrm{~g} \mathrm{~mL}^{-1}\right)$, the UV-vis absorption spectrum of hemoglobin solution was recorded and is shown in Figure 2. A variation of the oxidation state of $\mathrm{Fe}$ is known to produce a significant change in the observed spectra. $[33,34]$ The main oxidation states are Fe(III) known as methemoglobin (inactive form unable to bind oxygen) and $\mathrm{Fe}(\mathrm{II})$ (active form, able to bind oxygen to form a $\mathrm{Fe}(\mathrm{III}) \mathrm{O}_{2}{ }^{\cdot-}$ complex). These forms (see above) can be distinguished spectroscopically. The spectrum of hemoglobin used for subsequent dropcasts (black line) in Figure 2 corresponds to methemoglobin Fe(III) in accordance with the characteristic peak at $406 \mathrm{~nm}[33,34]$. In order to further confirm the hypothesis, the solution was treated with potassium cyanide $(\mathrm{KCN})$, which according to literature [9] leads to formation of stable FeCN complex with the Fe(III) oxidation state, since the $\mathrm{CN}^{-}$ligand binds preferentially to $\mathrm{Fe}(\mathrm{III})$. As a result a broad peak is observed at $\approx 530 \mathrm{~nm}$, consistent with literature reports. [35] 
Additionally to probe the other possible oxidation state $\mathrm{Fe}(\mathrm{II})$ the methemoglobin $\mathrm{Fe}(\mathrm{III})$ solution was treated with sodium dithionite $\mathrm{Na}_{2} \mathrm{~S}_{2} \mathrm{O}_{4}$ which leads to a reduction of $\mathrm{Fe}(\mathrm{III})+\mathrm{e}^{-} \longrightarrow \mathrm{Fe}(\mathrm{II})$. A color change from dark brown to pink was observed and the UV-vis spectrum reveals the appearance of two peaks in the $500 \mathrm{~nm}$ region, which is consistent with the formation of oxy- $\mathrm{Hb} \mathrm{Fe}(\mathrm{III}) \mathrm{O}_{2}{ }^{-{ }^{-}}$complex.

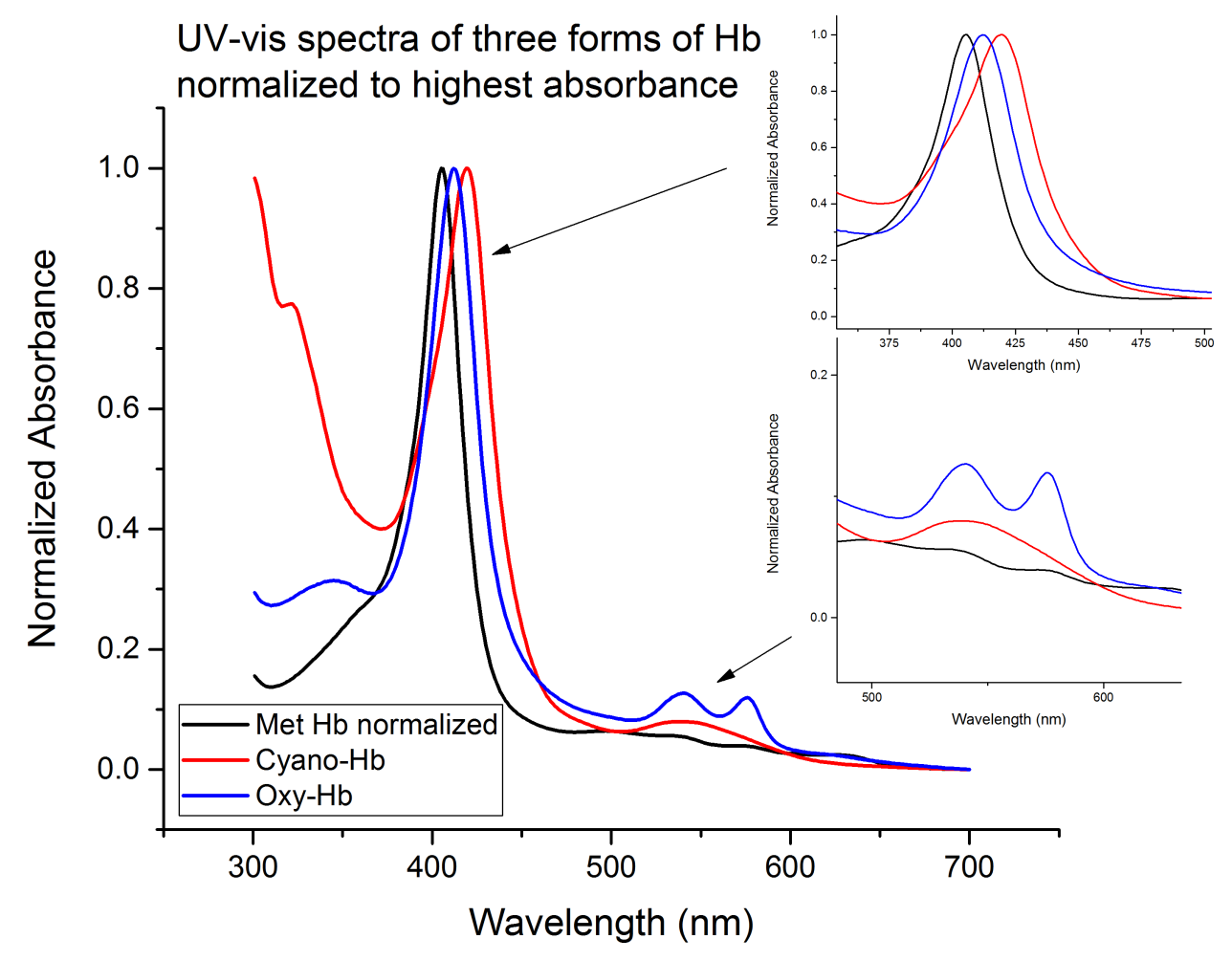

Figure 2: UV-vis absorption spectra of different oxidation states of $\mathrm{Hb}$

\subsection{Optical microscopy}

Dropcast films of Nafion hemoglobin were initially investigated using bright field optical microscopy via identical sample preparation to the electrode functionalization (Figure S1). It can be seen that hemoglobin forms aggregates and/or agglomerates. Further there is evidence of porous structure in the Nafion layers. The diameter of a single $\mathrm{Hb}$ molecule is approximately $5 \mathrm{~nm}$ [36] assuming a spheroidal shape. Nafion layers are known to possess a complex structure with structural heterogeneity on multiple spatial scales from 0.1 to $100 \mathrm{~nm}$ with a wide range of pore sizes and channel structure. $[37,38]$ As a result it is experimentally very difficult to define the exact location of hemoglobin within such layers as it may occupy some of the larger pores. Nevertheless resulting from the 3 step dropcasting procedure a large amount will remain embedded between the two Nafion matrices, within the 'sandwich'.

\subsection{Electrochemical Experiments}

\subsubsection{Effect of dissolved dioxygen}

The focus of the present work is the interaction of dioxygen with hemoglobin. The voltammetric response from Naf-Hb-Naf film was initially evaluated under two sets of conditions in order to deter- 


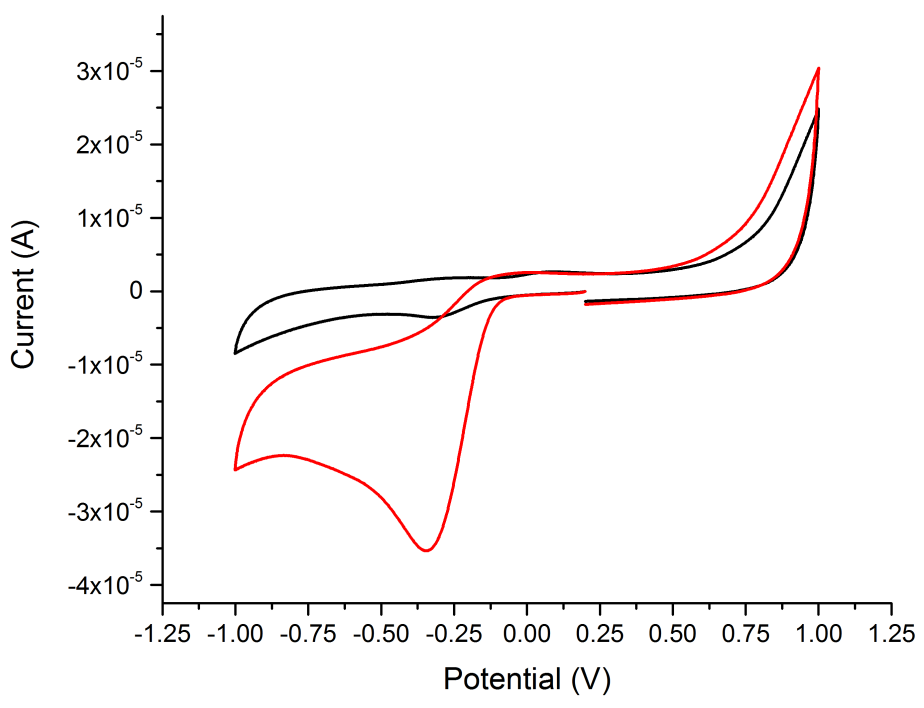

Figure 3: Effect of degassing on the observed current response for Naf-Hb-Naf modified electrode; black line degassed, red line non-degassed solution (PBS buffer $\mathrm{pH}$ 7.4). All potentials were measured against the SCE electrode (+0.250 V vs Standard Hydrogen Electrode). Scan rate: $250 \mathrm{mVs}^{-1}$.

mine the effect of oxygen presence in the solution: deaerated under nitrogen atmosphere and normal aerated (air saturated) conditions. Figure 3 shows the current response obtained under these two conditions, a peak is evidenced at $0.25 \mathrm{~V}$ vs SCE which is consistent with previous reports. [10] It is clear that under deaerated conditions virtually no oxygen reduction current response is observed (except for slight residual oxygen remaining after the degassing procedure), while aerated conditions provides a clear oxygen reduction response. Hence subsequent experiments were conducted using variable oxygen concentrations.

\subsubsection{Effect of Nafion thickness on the current response}

As outlined above Nafion ionomer was used to immobilize hemoglobin on the electrode surface. Initially the effect of Nafion modification was evaluated by adjusting the thickness of the Nafion layers and comparing the resultant response to the bare unmodified glassy carbon electrode in the absence of hemoglobin. The resultant cyclic voltammograms (CVs) are shown in Figure S2 and no significant deviations were observed in comparison to the bare glassy carbon electrode, which is consistent with previous literature reports of high Nafion porosity and gas permeability. [31,39]

\subsubsection{Effect of the hemoglobin concentration}

In order to test the hypothesis of the observed oxygen reduction rather than direct electrochemistry of $(F e(I I) / F e(I I I)$ couple) a variable amount of $\mathrm{Hb}$ was dropcast on a glassy carbon electrode. The results are shown in Figure S3. It can be seen that higher concentrations of dropcast hemoglobin leads to a decrease in current magnitude and is indicative of a partial blockage of the electrode. This is the evidence of the indirect nature of the hemoglobin reaction since as for direct $\mathrm{Hb}$ redox processes an increase in signal with increasing amount of $\mathrm{Hb}$ would be anticipated. 


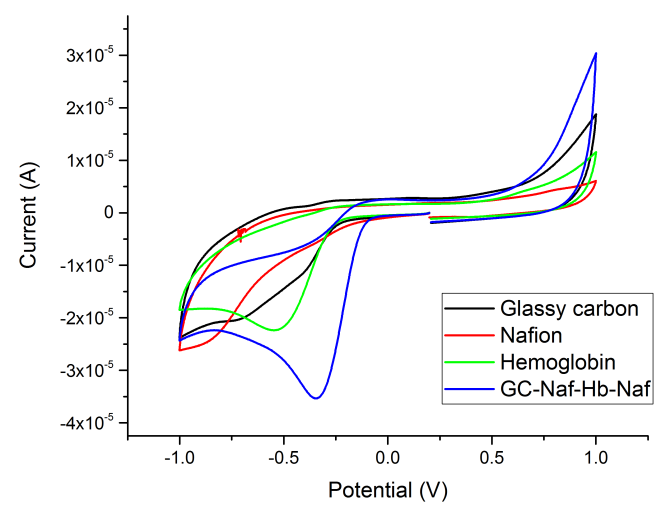

Figure 4: Effect of the modification procedure on the observed response in the air atmosphere for the oxygen reduction (PBS buffer $\mathrm{pH}$ 7.4). All potentials were measured against SCE electrode $(+0.250$ $\mathrm{V}$ vs Standard Hydrogen Electrode) Scan rate: $250 \mathrm{mVs}^{-1}$.

\subsubsection{Comparing the modified electrode responses}

Having established the impact of the presence of oxygen and the suitable thickness of Nafion and hemoglobin layers, the effect of electrode modification on the observed current response was next investigated. The comparison between bare glassy carbon, Nafion modified, hemoglobin and Nafion$\mathrm{Hb}-\mathrm{Nafion}$ electrode is given in Figure 4. For the unmodified glassy carbon electrode the peak potential was $-0.75 \mathrm{~V}$, the Nafion-modified peak potential was $-0.80 \mathrm{~V}$, hemoglobin-modified $-0.50 \mathrm{~V}$ and for the Naf-Hb-Naf electrode $-0.27 \mathrm{~V}$ (all potentials reported vs SCE). A clear potential shift to less negative potentials is observed for the hemoglobin-modified (green line) and Naf-Hb-Naf (blue line) electrode, which is indicative of oxygen-hemoglobin interaction. The largest potential shift is observed for the Naf-Hb-Naf electrode.

Further experiments involved saturating the solution with dioxygen. The comparison of oxygen saturated and normal aerated conditions for the Naf-Hb-Naf modified electrode is shown in Figure 5. The peak current potential is shifted to a more negative values at higher concentrations of oxygen. An inflexion (approximately $-0.275 \mathrm{~V}$ vs $\mathrm{SCE}$ ) is observed for the oxygen saturated solution in comparison to normal aerated conditions. The potential of the inflexion corresponds to the peak potential observed for the aerated solution. A comparison of all the electrode modifications is shown in Figure 6. For the unmodified glassy carbon electrode the peak potential was $-0.75 \mathrm{~V}$, the Nafion-modified peak potential was $-0.80 \mathrm{~V}$, that of hemoglobin-modified $-0.75 \mathrm{~V}$ and for the Naf-Hb-Naf electrode $-0.51 \mathrm{~V}$ (all potentials reported vs SCE).

\subsubsection{Hemin Cyclic Voltammetry}

In order to compare the response of hemoglobin and determine the effect of the surrounding protein, the observed response was compared to a Hemin modified electrode in air atmosphere (see supplementary Figure S4). Similar ORR catalytic activity was observed for the Hemin modified electrode. The similarity of the observed responses for the Hemoglobin and Hemin electrodes emphasises that it is the iron active site that plays a key role for the catalytic response.

\subsubsection{Scan rate study}

It is important to consider the nature of the observed process and in particular distinguish between surface bound or diffusional processes. For that purpose a scan rate study was conducted. Figure 7 


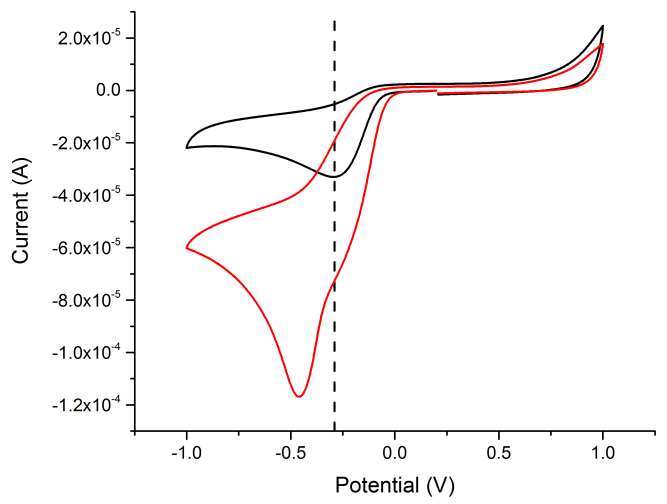

Figure 5: Comparison between voltamograms measured for the Naf-Hb-Naf electrode in air atmosphere (black line) and under a pure oxygen atmosphere (red line) at a scan rate $250 \mathrm{mV} / \mathrm{s}$. All potentials were measured against the SCE electrode $(+0.250 \mathrm{~V}$ vs Standard Hydrogen Electrode)

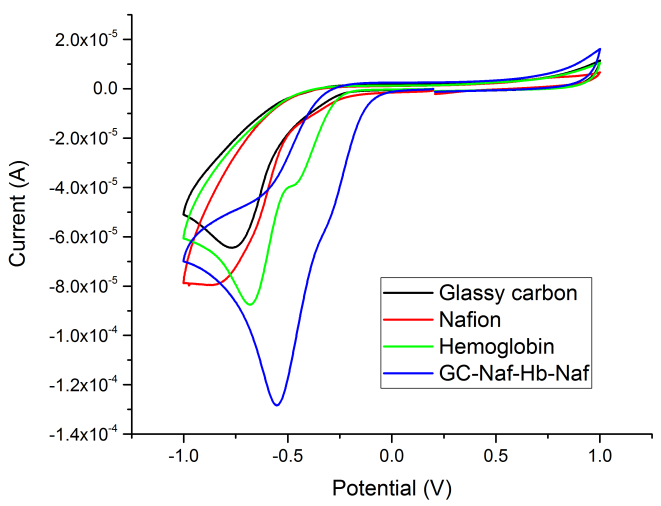

Figure 6: Effect of the electrode modification procedure on the observed response in a pure oxygen atmosphere (PBS buffer $\mathrm{pH} 7.4)$. All potentials were measured against SCE electrode $(+0.250 \mathrm{~V}$ vs Standard Hydrogen Electrode) at a scan rate of $250 \mathrm{mV} / \mathrm{s}$. 


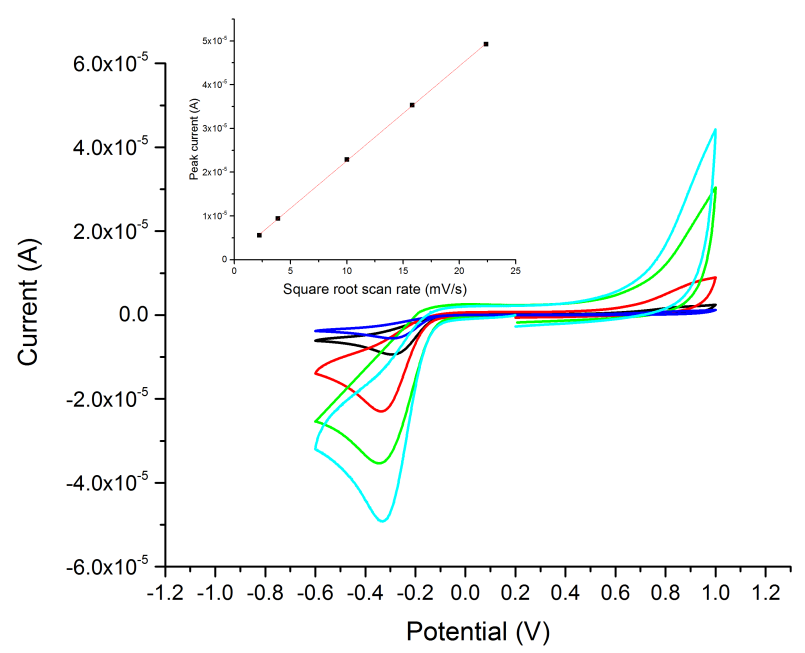

Figure 7: Effect of the scan rate on the observed current response for Naf-Hb-Naf electrode, measurements conducted under air atmosphere (PBS buffer $\mathrm{pH}$ 7.4). All potentials were measured against $\mathrm{SCE}$ electrode $(+0.250 \mathrm{~V}$ vs Standard Hydrogen Electrode) at a scan rate of $250 \mathrm{mV} / \mathrm{s}$.

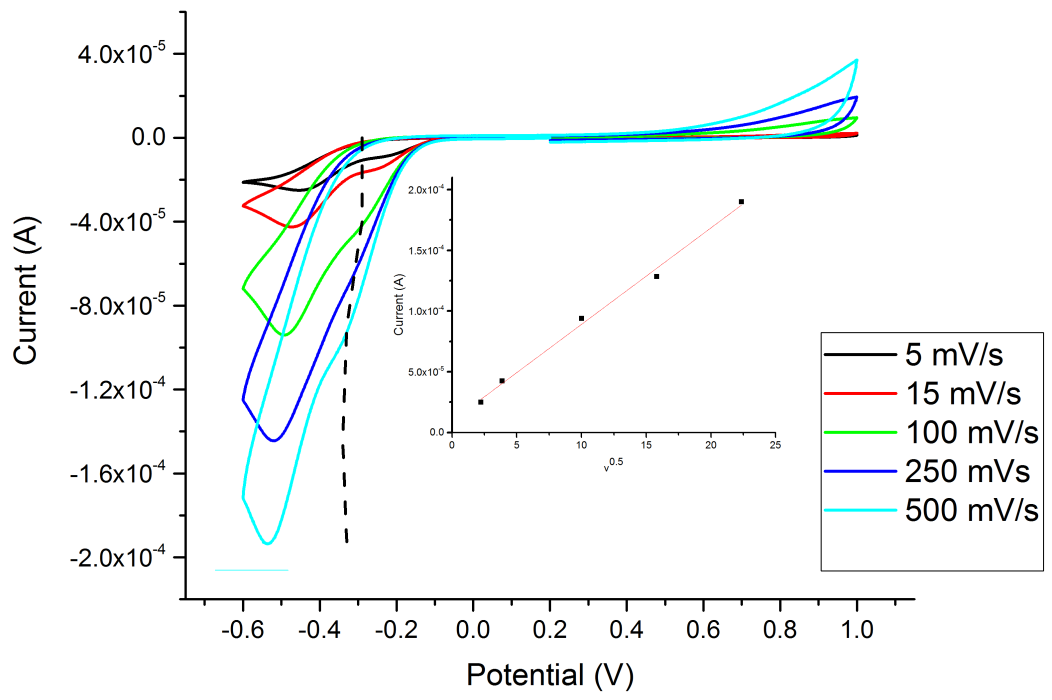

Figure 8: Voltammetric scan rate study of a pure oxygen saturated solution on Naf-Hb-Naf electrode (PBS buffer $\mathrm{pH} 7.4)$. All potentials were measured against SCE electrode $(+0.250 \mathrm{~V}$ vs Standard Hydrogen Electrode) 
and Figure 8 show the observed current response with variable scan rate for the aerated and oxygen saturated cases. A plot of the peak current versus the square root of the scan rate is clearly linear with a zero intercept for both cases, which is indicative of a diffusional process taking place. [40,41] This is a further evidence that indirect oxygen-hemoglobin interaction is being observed as for the surface bound film linear peak current vs scan rate dependence would be anticipated. In Section 3.5, the peak current magnitude will be compared with the response predicted for an unmodified glassy carbon electrode.

\subsection{Tafel Plots}

In order to gain insights about the electron transfer process for the modified electrodes, Tafel plots were plotted for unmodified (Figure S5), Nafion-modified (Figure S6), Hb modified (Figure S7) and Naf-Hb-Naf modified electrodes (Figure S8). The averaged value over three measurements at a scan rate of $250 \mathrm{mV} / \mathrm{s}$ were recorded and the errors estimated. Transfer coefficient $[42,43], \alpha$, for unmodified glassy carbon and Nafion modified electrode were $0.30 \pm 0.05$ and $0.23 \pm 0.04$ respectively, consistent with previous literature reports. [44,45] For the hemoglobin modified electrode an $\alpha$ value of $0.35 \pm 0.05$ was observed. The highest $\alpha$ was observed for Nafion-Hb-Nafion modified electrode of $0.57 \pm 0.07$. This is indicative of an altered transition state for the irreversible heterogeneous electron transfer process in which in all cases the initial electron transfer is evidently rate-determining.

\subsection{Comparison of experiment with theoretical predictions}

Having established the nature of the signal is due to dissolved oxygen further insights can be gained through theoretical predictions of the current response through the use of the Randles-Ševčík equation (Equation 4). [41]

$$
I_{\text {peak }}=2.99 \times 10^{5} n\left(n^{*}+\alpha\right)^{1 / 2} A D^{1 / 2} C v^{1 / 2}
$$

where $\mathrm{n}$ is the overall number of electrons transferred, $n^{*}$ is the number of electrons involved prior to the rate determining step, $\alpha$ is the transfer coefficient, $\mathrm{A}$ is the area of the electrode, $\mathrm{D}$ is the diffusion coefficient of oxygen, $\mathrm{C}$ is the concentration of oxygen in bulk solution and the temperature is $25.0 \pm 0.1 C^{\circ}$. In the present work $n^{*}$ denotes the number of electrons transferred before the rate determining step. The first electron transfer to $\mathrm{O}_{2}$ is rate determining hence, the preceding number of electrons transferred is zero and the assumption was used for subsequent calculations. Note that this approach follows the IUPAC $[42,43]$ nomenclature for Tafel analysis and updates the n $\alpha$ approach which appears for example in the first edition of the Bard and Faulkner Electrochemical Methods Fundamentals and Applications textbook. [41]

\subsubsection{Aerated atmosphere}

Figure 9 shows the experimental results (scatter points) and theoretical predictions (dashed line) relating to a solution equilibrated with air based on the following parameters: concentration of oxygen $0.26 \mathrm{mM}$, [46] $A=7.3 \times 10^{-6} \mathrm{~m}^{2}, D_{\text {oxygen }}=1.96 \times 10^{-9} \mathrm{~m}^{2} \mathrm{~s}^{-1}[44,47], n^{*}=0$. Different transfer coefficient values $(\alpha=0.26$ and $\alpha=0.57)$ and number of electrons transferred $(n=2$ and $n=4)$ were used to simulate the different reaction mechanisms (Reaction 1 and Reaction 2). It can be seen that for the Nafion modified electrode excellent agreement is observed with the theoretical prediction for the bare glassy carbon electrode and Reaction 1 leading to formation of hydrogen peroxide and overall two electron transfer and the Nafion modified electrode. Good agreement was observed between the unmodified glassy carbon electrode in accordance with previous observation of the permeability of the Nafion film to oxygen. [31,39] The current at the Naf-Hb-Naf modified electrode significantly exceeds the peak current predicted for the two electron oxygen reduction. This is indicative of a changed reaction pathway. The best agreement is observed for a four electron transfer and the transfer coefficient value of 0.57 , the latter corresponding with the experimental value (section 3.4). 


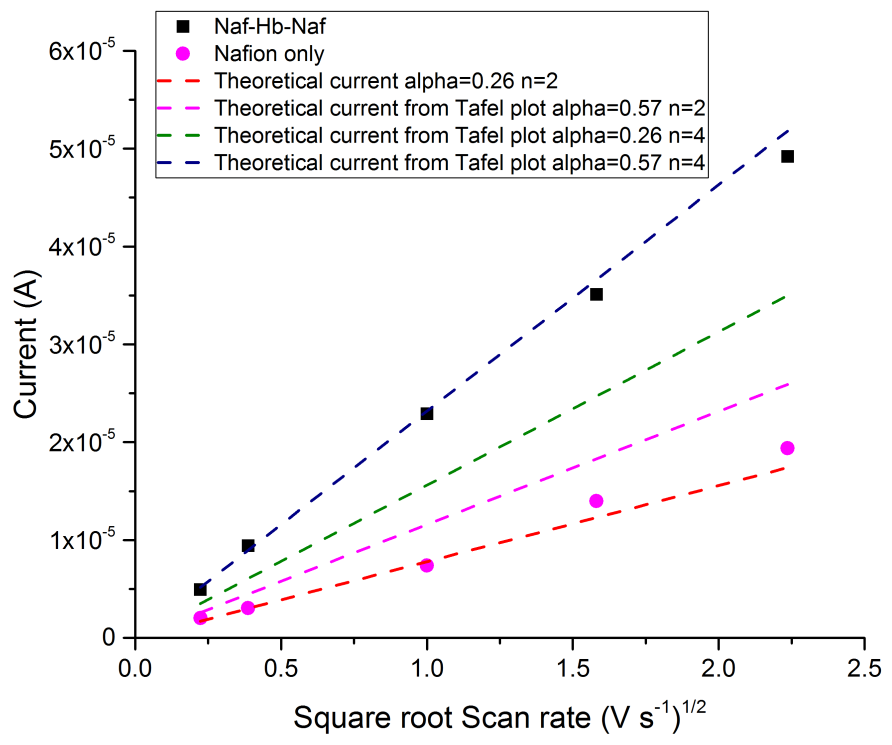

Figure 9: Comparison between experimentally observed currents and theoretical predictions by Randles-Š evč́́k equation under air atmosphere (PBS buffer $\mathrm{pH}$ 7.4)

\subsubsection{Oxygen atmosphere}

Having observed the behaviour under an air atmosphere, the oxygen saturated solution was analyzed. Figure 10 shows the theoretical predictions (indicated by label "theoretical" dashed line)for the oxygen saturated solution case. The following parameters were used for all theoretical calculations: $C_{\mathrm{O}_{2}}=$ $1.26 \mathrm{mM}$, [46] $A=7.3 \times 10^{-6} \mathrm{~m}^{2}, D_{\text {oxygen }}=1.96 \times 10^{-9} \mathrm{~m}^{2} \mathrm{~s}^{-1},[44,47] n^{*}=0$. Different transfer coefficient values $(\alpha=0.26$ and $\alpha=0.57)$ and number of electrons transferred $(n=2$ and $n=4)$ were used to simulate the different reaction mechanisms. For the Nafion functionalised electrode excellent agreement is observed to the theoretical prediction for a bare glassy carbon electrode $(\mathrm{n}=2, \alpha=0.26)$, this indicates that Nafion does not provide a diffusional barrier for the dissolved oxygen and it is likely that Reaction 1 takes place. It can be seen that a significantly higher current magnitude for the Naf$\mathrm{Hb}-\mathrm{Naf}$ modified electrode is observed in comparison to the theoretical prediction based on Reaction 1 which leads to formation of hydrogen peroxide. The observed experimental current is most consistent with a four electron transfer process and the transfer coefficient value of 0.57 which corresponds to the Reaction 2. The experimental current magnitude is a little lower in comparison to the theoretical prediction, which could be possibly explained by the fact that some of the oxygen formed by the breakdown of hydrogen peroxide may diffuse away resulting in a lowered current response. 


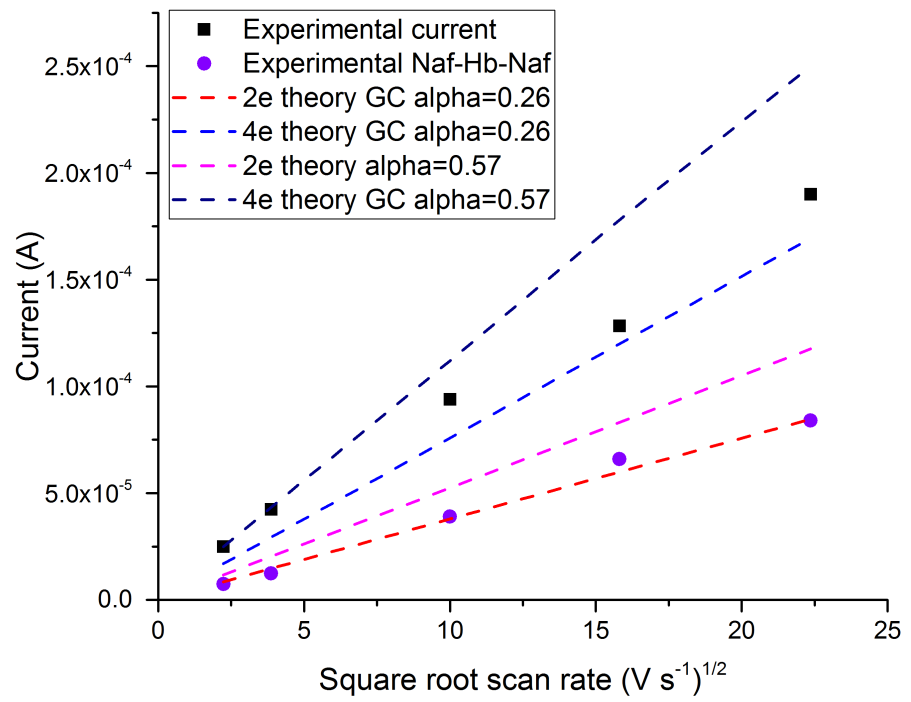

Figure 10: Comparison between experimentally observed currents and theoretical predictions by Randles-Ševčík equation under pure saturated oxygen atmosphere (PBS buffer pH 7.4)

\subsubsection{Hemoglobin hydrogen peroxide reactions}

It has been suggested in the literature [48] that $\mathrm{Hb}$ can react with the $\mathrm{H}_{2} \mathrm{O}_{2}$ formed by electrochemical oxygen reduction and initiate a catalytic cycle. In order to test this hypothesis hydrogen peroxide $(c=5 \mathrm{mM})$ was added to the degassed solution (nitrogen bubbled for $20 \mathrm{~min}$ ) and the resultant process was investigated through a scan rate study. The behaviour appears to be diffusionally limited in accordance with current against the square root of scan rate plot giving a straight line and is shown in Figure 11. Note: no hydrogen peroxide reduction was observed in the potential window shown at an unmodified glassy carbon electrode due to slow kinetics significantly higher overpotential is required. [49] According to literature [22] the following reaction may take place in the presence of hemoglobin:

$$
\mathrm{Fe}(\mathrm{III}) \mathrm{O}_{2}{ }^{\cdot-}+\mathrm{H}_{2} \mathrm{O}_{2} \longrightarrow \mathrm{Fe}(\mathrm{IV}) \mathrm{O}^{--}+\mathrm{O}_{2}+\mathrm{H}_{2} \mathrm{O}
$$

It is evident that Naf-Hb-Naf is able to react with hydrogen peroxide and as a result this reaction is likely to contribute to the observed enhanced current response for the case of oxygen reduction. From the results above, a peroxidase reactivity of haemoglobin has been shown using voltammetricaly. This activity is known to occur naturally within the red blood cells, but is masked by the more efficient activity of catalase and glutathione peroxidase. [50]

\subsection{Proposed Mechanism of ORR on Naf-Hb-Naf electrode}

Having investigated the current response of Naf-Hb-Naf films under variable oxygen concentrations it is necessary to account for the observed current magnitudes. It is clear that presence of Hemoglobin leads to an altered reaction pathway for the oxygen reduction. Through combination of the obtained experimental data and previous literature reports $[21,48,51]$ we propose the following mechanism for the oxygen reduction at Naf-Hb-Naf modified electrode. Oxygen is known to permeate the Nafion membranes and so can be reduced at the electrode surface. As a result the proposed mechanism does not require hemoglobin to be in electrical contact with the glassy carbon electrode surface. Indeed the Nafion layer between the electrode and the hemoglobin layer likely prevents this. At the 


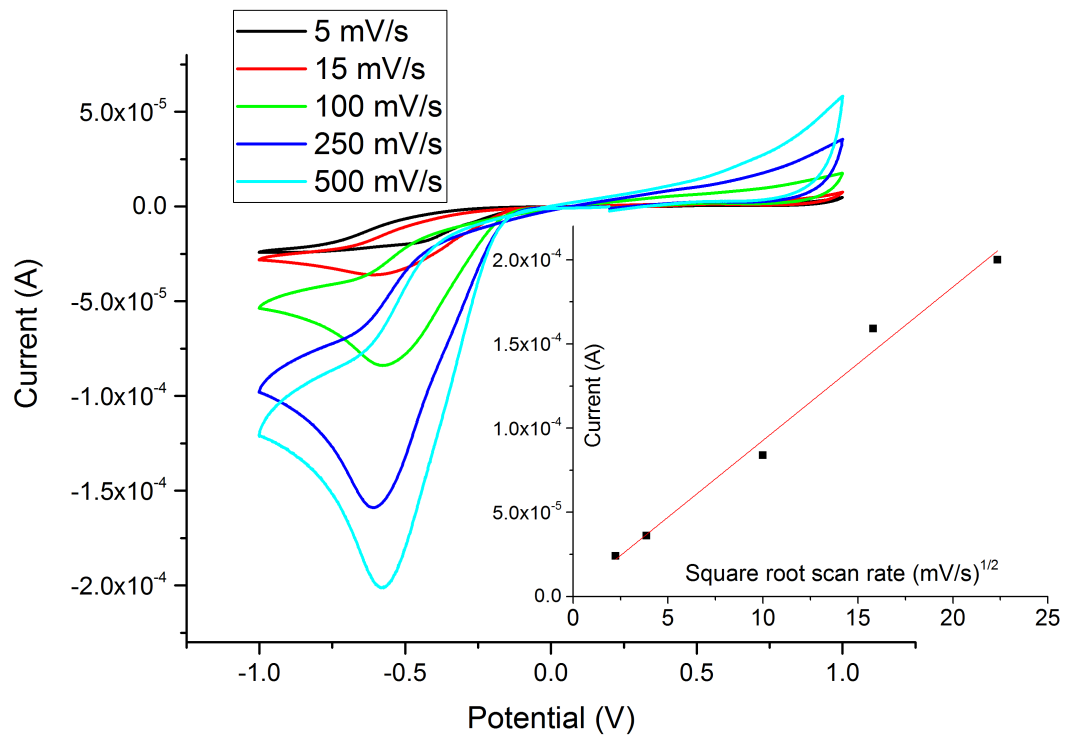

Figure 11: Scan rate study of degassed $5 \mathrm{mM}$ hydrogen peroxide solution on Naf-Hb-Naf electrode (PBS buffer $\mathrm{pH} 7.4)$. All potentials were measured against SCE electrode $(+0.250 \mathrm{~V}$ vs Standard Hydrogen Electrode)

surface of glassy carbon electrode dioxygen superoxide radical $\left(\mathrm{O}_{2}{ }^{\cdot-}\right)$ is generated (Eq. 5) electron transfer from the GC electrode. Hemoglobin adjacent to the electrode may likely provide adsorption sites for the dioxygen superoxide radical $\left(\mathrm{O}_{2}{ }^{--}\right)$as a result of the stabilization effect the potential for the reduction is shifted positively compared to the $\mathrm{O}_{2} / \mathrm{O}_{2}{ }^{--}$couple. Possible further steps of the mechanism involve disproportionation [52] of the generated superoxide species into hydrogen peroxide and oxygen species followed by subsequent reaction leading to a formation of a range of hemoglobin complexes. The resultant activated complex is equivalent to oxyhemoglobin and can participate in subsequent reactions. It is worth noting that the formation of the $\mathrm{Fe}(\mathrm{III}) \mathrm{O}_{2}{ }^{\cdot-}$ species has been clearly demonstrated previously using spectroelectrochemistry on various electrodes. [53,54] The Fe(III) bound dioxygen superoxide accepts a second electron from the superoxide species and this leads to formation of hydrogen peroxide (Eq. 7). The formed hydrogen peroxide that is in close proximity to the electrode can participate in further enzymatic reactions resulting in catalytic oxygen formation and subsequent oxygen reduction at the electrode (Eq. 8). It is not possible to further probe the intermediates due to the fast rates of the reaction (Eq.9-10). The proposed mechanism is consistent with an overall four electron reduction

$$
\begin{gathered}
\mathrm{O}_{2}+\mathrm{e}_{\text {electrode }}^{-} \longrightarrow \mathrm{O}_{2}^{\cdot-} \\
\mathrm{Fe}(\mathrm{III})+\mathrm{O}_{2}^{\cdot-} \longrightarrow \mathrm{Fe}(\mathrm{III}) \mathrm{O}_{2}^{\cdot-} \\
\mathrm{Fe}(\mathrm{III}) \mathrm{O}_{2}{ }^{--}+\mathrm{e}_{\mathrm{O}_{2}^{--}}^{-}+2 \mathrm{H}^{+} \longrightarrow \mathrm{Fe}(\mathrm{II})+\mathrm{H}_{2} \mathrm{O}_{2}+\mathrm{O}_{2} \\
\mathrm{Fe}(\mathrm{III}) \mathrm{O}_{2}^{\cdot-}+\mathrm{H}_{2} \mathrm{O}_{2} \longrightarrow \mathrm{Fe}(\mathrm{IV}) \mathrm{O}+\mathrm{H}_{2} \mathrm{O}+\mathrm{O}_{2} \\
\mathrm{Fe}(\mathrm{III})+\mathrm{H}_{2} \mathrm{O}_{2} \longrightarrow \mathrm{Fe}(\mathrm{IV}) \mathrm{O}^{\cdot}+\mathrm{H}_{2} \mathrm{O} \\
\mathrm{Fe}(\mathrm{IV}) \mathrm{O}^{\cdot}+\mathrm{H}_{2} \mathrm{O}_{2} \longrightarrow \mathrm{Fe}(\mathrm{III})+\mathrm{O}_{2}+\mathrm{H}_{2} \mathrm{O}
\end{gathered}
$$




\section{Conclusions}

ORR on the Naf-Hb-Naf modified electrode proceeds via a four electron pathway through catalytic reduction of hydrogen peroxide. The disproportionation of hydrogen peroxide produced by oxygen reduction reaction at the electrode by trapped hemoglobin leads to an increase in the current output and leads to a significant catalytic efficiency. The highest amplification is observed for the layered structure, which can be potentially ascribed to beneficial effect of Nafion leading to favourable conformation of trapped hemoglobin, evidenced by a change in transfer coefficient value. In addition a clear demonstration of the effect of the dissolved oxygen on the resultant current response is shown. The Nafion-protein layer was investigated in a solution under various oxygen concentrations or hydrogen peroxide concentrations. From the experimentally observed peak currents, a mechanism for the catalytic reaction on a glassy carbon electrode is elucidated. The work highlights the need for an efficient support for the catalyst which in the present case is provided by the Nafion layer structure. The methodological approach taken may have generic value.

\section{Acknowledgement}

We acknowledge funding from the European Research Council (ERC) under the European Union's Seventh Frame-work Program (FP/2007-2013)/ERC Grant Agreement no. [320403].

\section{References}

[1] D. Li, H. Lv, Y. Kang, N. M. Markovic, and V. R. Stamenkovic, "Progress in the Development of Oxygen Reduction Reaction Catalysts for Low-Temperature Fuel Cells*," Annu. Rev. Chem. Biomol. Eng., vol. 7, no. 1, pp. 509-532, 2016.

[2] Q. He and E. J. Cairns, "ReviewRecent Progress in Electrocatalysts for Oxygen Reduction Suitable for Alkaline Anion Exchange Membrane Fuel Cells," J. Electrochem. Soc., vol. 162, no. 14, pp. F1504-F1539, 2015.

[3] B. Šljukić, C. E. Banks, and R. G. Compton, "An overview of the electrochemical reduction of oxygen at carbon-based modified electrodes," J. Iran. Chem. Soc., vol. 2, no. 1, pp. 1-25, 2005.

[4] M. F. Perutz, A. J. Wilkinson, M. Paoli, and G. G. Dodson, "The Stereochemical Mechanism Of The Cooperative Effects In Hemoglobin Revisited," Annu. Rev. Biophys. Biomol. Struct., vol. 27, no. 1, pp. 1-34, 1998.

[5] I. Lalezari, P. Lalezari, C. Poyart, M. Marden, J. Kister, B. Bohn, G. Fermi, and M. F. Perutz, "New effectors of human hemoglobin: structure and function," Biochemistry, vol. 29, no. 6, pp. 1515-1523, 1990.

[6] J. P. Collman and L. Fu, "Synthetic Models for Hemoglobin and Myoglobin," Acc. Chem. Res., vol. 32, no. 6, pp. 455-463, 1999.

[7] M. Katterle, U. Wollenberger, and F. W. Scheller, "Electrochemistry of hemoglobin at modified silver electrodes is not a redox-process of iron protoporphyrin IX," Electroanalysis, vol. 9, no. 18, pp. 1393-1396, 1997.

[8] M. Baccarin, B. C. Janegitz, R. Berté, F. C. Vicentini, C. E. Banks, O. Fatibello-Filho, and V. Zucolotto, "Direct electrochemistry of hemoglobin and biosensing for hydrogen peroxide using a film containing silver nanoparticles and poly(amidoamine) dendrimer," Mater. Sci. Eng. C, vol. 58, pp. 97-102, 2016. 
[9] F. W. Scheller, N. Bistolas, S. Liu, M. Jänchen, M. Katterle, and U. Wollenberger, "Thirty years of haemoglobin electrochemistry.," Adv. Colloid Interface Sci., vol. 116, no. 1-3, pp. 111-20, 2005.

[10] R. J. Toh, W. K. Peng, J. Han, and M. Pumera, "Direct In Vivo Electrochemical Detection of Haemoglobin in Red Blood Cells," Sci. Rep., vol. 4, p. 6209, 2014.

[11] S. Treimer, A. Tang, and D. C. Johnson, "A Consideration of the Application of Koutecký-Levich Plots in the Diagnoses of Charge-Transfer Mechanisms at Rotated Disk Electrodes," Electroanalysis, vol. 14, no. 3, pp. 165-171, 2002.

[12] R. Zhou, Y. Zheng, M. Jaroniec, and S.-Z. Qiao, "Determination of the Electron Transfer Number for the Oxygen Reduction Reaction: From Theory to Experiment," ACS Catal., vol. 6, no. 7, pp. 4720-4728, 2016.

[13] J. Masa, C. Batchelor-McAuley, W. Schuhmann, and R. G. Compton, "Koutecky-Levich analysis applied to nanoparticle modified rotating disk electrodes: Electrocatalysis or misinterpretation," Nano Res., vol. 7, no. 1, pp. 71-78, 2013.

[14] A. Frumkin and L. Nekrasov, "No Title," Dokl. Akad. Nauk SSSR, vol. 126, p. 115, 1959.

[15] H. K. Kuiken, E. P. A. M. Bakkers, H. Ligthart, and J. J. Kelly, "The Rotating Ring-Ring Electrode. Theory and Experiment," J. Electrochem. Soc., vol. 147, no. 3, pp. 1110-1116, 2000.

[16] M. T. Gladwin, J. H. Crawford, and R. P. Patel, "The biochemistry of nitric oxide, nitrite, and hemoglobin: role in blood flow regulation," Free Radic. Biol. Med., vol. 36, no. 6, pp. 707-717, 2004.

[17] A. Hayashi, T. Suzuki, and M. Shin, "An enzymic reduction system for metmyoglobin and methemoglobin, and its application to functional studies of oxygen carriers," Biochim. Biophys. Acta Protein Struct., vol. 310, no. 2, pp. 309-316, 1973.

[18] E. Hegesh, N. Gruener, S. Cohen, R. Bochkovsky, and H. Shuval, "A sensitive micromethod for the determination of methemoglobin in blood," Clin. Chim. Acta, vol. 30, no. 3, pp. 679-682, 1970.

[19] J. B. Wittenberg, B. A. Wittenberg, J. Peisach, and W. E. Blumberg, "On the state of the iron and the nature of the ligand in oxyhemoglobin.," Proc. Natl. Acad. Sci. U. S. A., vol. 67, no. 4, pp. 1846-1853, 1970.

[20] H. Chen, M. Ikeda-Saito, and S. Shaik, "Nature of the FeO 2 Bonding in Oxy-Myoglobin: Effect of the Protein," J. Am. Chem. Soc., vol. 130, no. 44, pp. 14778-14790, 2008.

[21] C. C. Winterbourn, B. M. McGrath, and R. W. Carrell, "Reactions involving superoxide and normal and unstable haemoglobins.," Biochem. J., vol. 155, no. 3, pp. 493-502, 1976.

[22] J. Everse and N. Hsia, "The toxicities of native and modified hemoglobins," Free Radic. Biol. Med., vol. 22, no. 6, pp. 1075-1099, 1997.

[23] A. Salimi, E. Sharifi, A. Noorbakhsh, and S. Soltanian, "Direct voltammetry and electrocatalytic properties of hemoglobin immobilized on a glassy carbon electrode modified with nickel oxide nanoparticles," Electrochem. commun., vol. 8, no. 9, pp. 1499-1508, 2006.

[24] K. Amreen and A. S. Kumar, "Electrochemical redox signaling of hemoglobin in human whole blood and its relevance to anemia and thalassemia diagnosis.," Analyst, vol. 141, no. 7, pp. 2145$2149,2016$.

[25] Z. Dai, S. Liu, H. Ju, and H. Chen, "Direct electron transfer and enzymatic activity of hemoglobin in a hexagonal mesoporous silica matrix," Biosens. Bioelectron., vol. 19, no. 8, pp. 861-867, 2004. 
[26] X. Han, W. Cheng, Z. Zhang, S. Dong, and E. Wang, "Direct electron transfer between hemoglobin and a glassy carbon electrode facilitated by lipid-protected gold nanoparticles," Biochim. Biophys. Acta - Bioenerg., vol. 1556, no. 2-3, pp. 273-277, 2002.

[27] C. Lei, U. Wollenberger, N. Bistolas, A. Guiseppi-Elie, and F. Scheller, "Electron transfer of hemoglobin at electrodes modified with colloidal clay nanoparticles," Anal. Bioanal. Chem., vol. 372 , no. 2, pp. 235-239, 2002.

[28] H. F. Bunn, "Evolution of mammalian hemoglobin function.," Blood, vol. 58, no. 2, pp. 189-97, 1981.

[29] M. Shao, Q. Chang, J.-P. Dodelet, and R. Chenitz, "Recent Advances in Electrocatalysts for Oxygen Reduction Reaction," Chem. Rev., vol. 116, no. 6, pp. 3594-3657, 2016.

[30] H.-H. Yang and R. L. McCreery, "Elucidation of the Mechanism of Dioxygen Reduction on Metal-Free Carbon Electrodes," J. Electrochem. Soc., vol. 147, no. 9, pp. 3420-3428, 2000.

[31] J. Maruyama and I. Abe, "Cathodic oxygen reduction at the interface between Nafion $囚$ and electrochemically oxidized glassy carbon surfaces," J. Electroanal. Chem., vol. 527, no. 1-2, pp. 65-70, 2002.

[32] M. G. Banus, "A Design For A Saturated Calomel Electrode," Science (80-. )., vol. 93, no. 2425, pp. 601-602, 1941.

[33] Cruz-Landeira, M. J. Bal, O. Quintela, and M. Lopez-Rivadulla, "Determination of Methemoglobin and Total Hemoglobin in Toxicological Studies by Derivative Spectrophotometry," J. Anal. Toxicol., vol. 26, no. 2, pp. 67-72, 2002.

[34] J. Yi, G. Ye, L. M. Thomas, and G. B. Richter-Addo, "Degradation of human hemoglobin by organic C-nitroso compounds," Chem. Commun., vol. 49, no. 95, pp. 11179-11181, 2013.

[35] M. Zhu, R. Rodriguez, T. Wehr, and C. Siebert, "Capillary electrophoresis of hemoglobins and globin chains," J. Chromatogr. A, vol. 608, no. 1-2, pp. 225-237, 1992.

[36] S. Budavari, Merck Index. Royal Society of Chemistry, 12 ed., 1996.

[37] C.-H. Chia, Z. Wu, C.-H. Wu, R.-H. Cheng, and S. Ding, "Resolve the pore structure and dynamics of Nafion 117: application of high resolution 7Li solid state nuclear magnetic resonance spectroscopy," J. Mater. Chem., vol. 22, no. 42, pp. 22440-22445, 2012.

[38] K. A. Mauritz and R. B. Moore, "State of Understanding of Nafion," Chem. Rev., vol. 104, no. 10, pp. 4535-4586, 2004.

[39] D. R. Lawson, "Oxygen Reduction at Nafion Film-Coated Platinum Electrodes: Transport and Kinetics," J. Electrochem. Soc., vol. 135, no. 9, pp. 2247-2253, 1988.

[40] R. G. Compton and C. E. Banks, Understanding Voltammetry. Imperial College Press, 2nd revise ed., 2010.

[41] A. Bard and L. Faulkner, Electrochemical methods: fundamentals and applications. New York: Wiley, 2nd editio ed., 2001.

[42] R. Guidelli, R. G. Compton, J. M. Feliu, E. Gileadi, J. Lipkowski, W. Schmickler, and S. Trasatti, "Defining the transfer coefficient in electrochemistry: An assessment (IUPAC Technical Report)," Pure Appl. Chem., vol. 86, no. 2, pp. 245-258, 2014. 
[43] R. Guidelli, R. G. Compton, J. M. Feliu, E. Gileadi, J. Lipkowski, W. Schmickler, and S. Trasatti, "Definition of the transfer coefficient in electrochemistry (IUPAC Recommendations 2014)," Pure Appl. Chem., vol. 86, no. 2, pp. 259-262, 2014.

[44] R. Nissim and R. G. Compton, "Nonenzymatic Electrochemical Superoxide Sensor," ChemElectroChem, vol. 1, no. 4, pp. 763-771, 2014.

[45] G. Gotti, D. Evrard, K. Fajerwerg, and P. Gros, "Oxygen reduction reaction features in neutral media on glassy carbon electrode functionalized by chemically prepared gold nanoparticles," $J$. Solid State Electrochem., vol. 20, no. 6, pp. 1539-1550, 2016.

[46] R. Battino, "Oxygen and Ozone: Solubility Data Series," Pergamon Press, vol. 7, 1981.

[47] K. Shimizu, L. Sepunaru, and R. G. Compton, "Innovative catalyst design for the oxygen reduction reaction for fuel cells," Chem. Sci., vol. 7, no. 5, pp. 3364-3369, 2016.

[48] E. Nagababu and J. M. Rifkind, "Reaction of hydrogen peroxide with ferrylhemoglobin: Superoxide production and heme degradation," Biochemistry, vol. 39, no. 40, pp. 12503-12511, 2000 .

[49] J. A. Cox and R. K. Jaworski, "Voltammetric reduction and determination of hydrogen peroxide at an electrode modified with a film containing palladium and iridium," Anal. Chem., vol. 61, no. 19, pp. 2176-2178, 1989.

[50] E. Nagababu, F. J. Chrest, and J. M. Rifkind, "Hydrogen-peroxide-induced heme degradation in red blood cells: the protective roles of catalase and glutathione peroxidase," Biochim. Biophys. Acta - Gen. Subj., vol. 1620, no. 1, pp. 211-217, 2003.

[51] B. B. Hasinoff, "Kinetics of carbon monoxide and oxygen binding to hemoglobin in human red blood cell suspensions studied by laser flash photolysis," Biophys. Chem., vol. 13, no. 2, pp. 173$179,1981$.

[52] B. H. J. Bielski and A. O. Allen, "Mechanism of the disproportionation of superoxide radicals," J. Phys. Chem., vol. 81, no. 11, pp. 1048-1050, 1977.

[53] S. A. Dragan, K. W. Olsen, E. G. Moore, and A. Fitch, "Spectroelectrochemical study of hemoglobin A, alpha- and beta-fumarate crosslinked hemoglobins; implications to autoxidation reaction," Bioelectrochemistry, vol. 73, no. 1, pp. 55-63, 2008.

[54] C. H. Taboy, C. Bonaventura, and A. L. Crumbliss, "Anaerobic Oxidations of Myoglobin and Hemoglobin by Spectroelectrochemistry," pp. 187-209, 2002. 